\title{
Proceso combinado de tratamiento con ácido y pirólisis para la recuperación de fósforo y la obtención de sólidos adsorbentes a partir de digestato
}

\author{
M. Atienza-Martínez ${ }^{1}$, M. García ${ }^{1}$, J. Ábrego $^{1}$, G. Gea $^{1}$ \\ ${ }^{1}$ Grupo de Procesos Termoquímicos (GPT) \\ Instituto de Investigación en Ingeniería de Aragón (I3A) \\ Universidad de Zaragoza, Mariano Esquillor s/n, 50018, Zaragoza, Spain. \\ Tel. +34-976762707, e-mail: atienza@unizar.es
}

\section{Resumen}

En el presente trabajo se pretende valorizar digestato de purín de vacuno y otros residuos orgánicos mediante un proceso combinado de tratamiento con ácido y pirólisis con el fin de recuperar el fósforo $(\mathrm{P})$ presente y obtener un sólido que pueda emplearse como material adsorbente.

\section{Introducción}

El P es un nutriente esencial para los seres vivos. En el caso de los vegetales, para que los niveles tanto de $\mathrm{P}$ como de nitrógeno $(\mathrm{N})$ y potasio $(\mathrm{K})$ sean apropiados es necesario emplear fertilizantes. La mayor parte de la demanda de $\mathrm{P}$ se destina a la fabricación de fertilizantes [1]. La actual fuente de P, la roca fosfática, es un recurso no renovable y su disponibilidad así como su calidad están disminuyendo [2]. Se hace necesario por tanto encontrar fuentes alternativas de $\mathrm{P}$, como pueden ser los residuos de origen animal o vegetal. Una de las alternativas es la aplicación directa de estos residuos sobre los suelos, pero, debido a los problemas que esto puede conllevar, resulta interesante estudiar la recuperación del $\mathrm{P}$ para la fabricación de fertilizantes. En la actualidad existen ya algunos estudios sobre la extracción de $\mathrm{P}$ a partir de purines [3].

Por otro lado, los purines también pueden ser valorizados mediante procesos termoquímicos como por ejemplo la pirólisis, en la cual se obtiene un sólido carbonoso (llamado char), un líquido y gases no condensables. El char, que es el producto mayoritario, puede ser empleado como sólido adsorbente, ya que la pirólisis mejora propiedades como la porosidad. Para conseguir sólidos con una mayor superficie específica es necesario activar el material antes de someterlo a pirólisis. La activación puede llevarse a cabo empleando ácidos, bases o sales $[4,5]$. El proceso de activación en medio ácido para la obtención de sólidos adsorbentes mediante pirólisis presenta ciertas similitudes en cuanto a condiciones de operación con el de extracción de $\mathrm{P}$, también en medio ácido, por lo que la etapa de extracción de P podría servir a su vez como etapa de activación del material para su posterior pirólisis y obtención de un sólido adsorbente.

El objetivo concreto de este trabajo es estudiar el efecto que tiene, por un lado, la relación líquido:sólido (L:S) empleada en la etapa de extracción-activación tanto sobre el porcentaje de $\mathrm{P}$ que se puede extraer del digestato como sobre la superficie específica del sólido obtenido tras la pirólisis, y por otro el efecto de la temperatura de pirólisis sobre la superficie específica del sólido.

\section{Materiales y métodos}

Como materia prima se ha empleado un digestato procedente de una mezcla de purín vacuno y diversos residuos orgánicos, que han sido previamente pasteurizados y digeridos anaeróbicamente. El contenido en $\mathrm{P}$ de este material es del 1,4\%.

En primer lugar se han llevado a cabo experimentos de extracción-activación. En todos los casos la duración de esta etapa ha sido de $24 \mathrm{~h}$ y la concentración de ácido sulfúrico de $3 \mathrm{~mol} \cdot \mathrm{dm}^{-3}$. Se ha variado la relación L:S entre 5:1 y 10:1. La carga de ácido empleada ha sido siempre superior a la carga estequiométrica (12,5 kg de ácido por $\mathrm{kg}$ de $\mathrm{P}$ presente en el digestato). Se ha mezclado el digestato con la cantidad de ácido sulfúrico necesaria y se ha agitado durante el tiempo establecido, tras el cual se ha centrifugado la mezcla y se ha recuperado de forma separada el sobrenadante y el sólido. Se ha determinado el contenido en $\mathrm{P}$ del sobrenadante y se ha calculado el porcentaje de $\mathrm{P}$ extraído del digestato. A continuación, se ha secado la fracción sólida y se ha pirolizado en un reactor de lecho fijo a dos temperaturas distintas $\left(550{ }^{\circ} \mathrm{C}\right.$ y $\left.850{ }^{\circ} \mathrm{C}\right)$. Una vez finalizado el experimento de pirólisis, se ha determinado la superficie específica del char 
mediante el método BET. Para poder comparar los resultados, se han llevado a cabo también experimentos de pirólisis de digestato, sin etapa de extracción-activación, a ambas temperaturas.

\section{Resultados y discusión Experimentos de extracción-activación}

En cuanto al porcentaje de $\mathrm{P}$ extraído del digestato tras la etapa de extracción-activación, un aumento de la relación L:S desde 5:1 hasta 10:1 ha provocado un aumento del porcentaje de $\mathrm{P}$ que se puede extraer desde $60,8 \%$ hasta $67,4 \%$. Dado que la concentración de ácido es la misma en ambos casos, el aumento de la relación L:S implica una mayor carga de ácido ( $\mathrm{kg}$ de ácido por $\mathrm{kg}$ de $\mathrm{P}$ presente en el digestato), lo cual puede favorecer la extracción de P. Además, una mayor relación L:S también favorece la transferencia de materia.

\section{Experimentos de pirólisis}

La superficie específica BET del char obtenido bajo las diferentes condiciones de operación estudiadas ha variado entre $70 \mathrm{~m}^{2} \cdot \mathrm{g}^{-1}$ y $440 \mathrm{~m}^{2} \cdot \mathrm{g}^{-1}$ (ver Figura 1). Para las dos temperaturas de pirólisis con las que se ha trabajado $\left(550{ }^{\circ} \mathrm{C}\right.$ y $\left.850{ }^{\circ} \mathrm{C}\right)$, la etapa de extracción-activación ha provocado un aumento de la superficie específica del char de pirólisis, si se compara con el digestato sin tratamiento con ácido, aunque la relación L:S no ha tenido efecto sobre esta propiedad del sólido. En el caso en el que no ha habido etapa de extracción-activación y en el que se ha empleado una relación L:S de 5:1 la temperatura de pirólisis no ha tenido efecto sobre la superficie específica BET, mientras que cuando la relación L:S empleada ha sido de 10:1 un aumento de temperatura desde $550{ }^{\circ} \mathrm{C}$ hasta $850{ }^{\circ} \mathrm{C}$ ha provocado un ligero aumento de esta propiedad del char.

\section{Conclusiones}

El proceso estudiado en el presente trabajo permite la conjunta extracción de $\mathrm{P}$ y la obtención de sólidos adsorbentes a partir de un residuo como es el digestato, mediante la combinación de una etapa de extracción-activación y otra de pirólisis. Bajo las condiciones de operación empleadas, ha sido posible extraer más del $60 \%$ del $\mathrm{P}$ presente en el digestato y obtener sólidos con una superficie específica BET superior a $400 \mathrm{~m}^{2} \cdot \mathrm{g}^{-1}$. Para una determinada temperatura de pirólisis, la superficie específica BET del char es mayor cuando el digestato ha sido sometido previamente a la etapa de extracciónactivación. Dentro de los intervalos estudiados, las condiciones de operación que favorecerían al mismo tiempo la extracción de $\mathrm{P}$ y la obtención de un char con elevada superficie específica BET serían una relación L:S de 10:1 y una temperatura de pirólisis de $850{ }^{\circ} \mathrm{C}$.

\section{REFERENCIAS}

[1]. CORDELL, D., DRANGERT, J.-O., and WHITE, S. The story of phosphorus: Global food security and food for thought. Global Environmental Change. 2009, 19(2), 292-305. Available from: doi:10.1016/j.gloenvcha.2008.10.009.

[2]. DONATEllo, S., TONG, D., and ChEESEMAN, C. R. Production of technical grade phosphoric acid from incinerator sewage sludge ash (ISSA). Waste Management. 2010, 30(8-9), 1634-1642. Available from: doi:10.1016/j.wasman.2010.04.009.

[3]. SzÖGI, A. A., VANOTTI, M. B., and HUNT, P. G. Phosphorus recovery from pig manure solids prior to land application. Journal of Environmental Management. 2015, 1-7. Available from: doi:10.1016/j.jenvman.2015.04.010

[4]. ZHANG, F.-S., NRIAGU, J. O., and ITOH, H. Mercury removal from water using activated carbons derived from organic sewage sludge. Water Research. 2005, 39(2-3), 389-395. Available from: doi:10.1016/j.watres.2004.09.027

[5]. MOnSAlvo, V. M., MOHEDANO, A. F., and RODRIGUEZ, J. J. Activated carbons from sewage sludge: Application to aqueous-phase adsorption of 4chlorophenol. Desalination. 2011, 277(1-3), 377-382. Available from: doi:10.1016/j.desal.2011.04.059

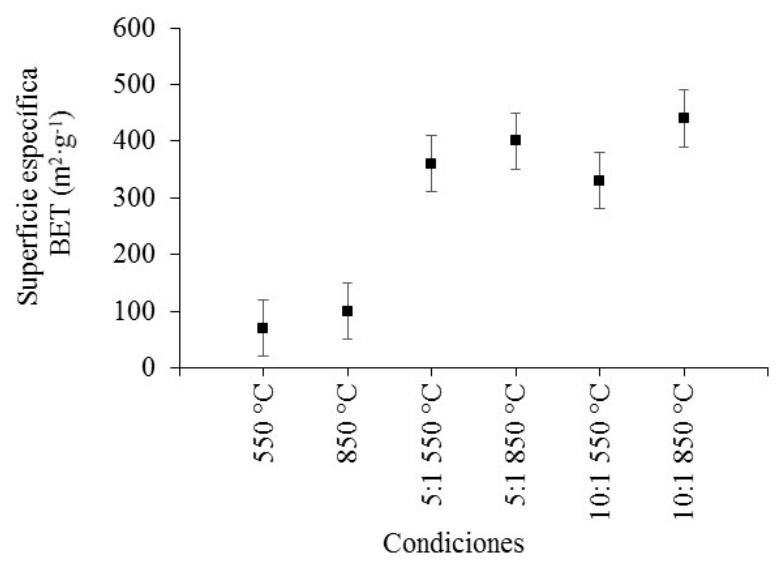

Figura 1. Superficie específica BET del char obtenido bajo diferentes condiciones. 